\title{
Taonga Māori in the British Museum de Dorota C.
} STARZECKA et al.

\section{Gilles Bounoure}

\section{OpenEdition}

\section{Journals}

\section{Édition électronique}

URL : http://journals.openedition.org/jso/6748

DOI : $10.4000 /$ jso.6748

ISSN : $1760-7256$

Éditeur

Société des océanistes

Édition imprimée

Date de publication : 31 décembre 2012

Pagination : 280-282

ISBN : 978-2-85430-033-8

ISSN : 0300-953x

Référence électronique

Gilles Bounoure, "Taonga Māori in the British Museum de Dorota C. starzecka et al. », Journal de la Société des Océanistes [En ligne], 135 | 2012-2, mis en ligne le 18 février 2013, consulté le 24 septembre 2020. URL : http://journals.openedition.org/jso/6748; DOI : https://doi.org/10.4000/jso 6748 
que dans la conception que se faisaient les colons européens de l'art māori. Les deux suivants (pp. 146178) décrivent la sculpture māori sous l'angle de la culture matérielle, depuis ses outils et leur mise en Suvre jusqu'à ses réalisations les plus emblématiques, et du fait de leur portée générale et de leur profondeur, l'ensemble de ces pages mérite d'être connu de tous les spécialistes des arts du Pacifique. Leur succèdent trois chapitres $(13,14,15$, pp. 179-257) examinant et comparant, d'après ce qui subsiste des sculptures Tarawhai, ce qu'elles doivent à leurs commanditaires d'abord māori, puis européens, missionnaires ou fonctionnaires, et enfin touristes attirés un temps par les ressources thermales de Rotorua et de ses environs.

S'appuyant sur l'étude d'œuvres «individuelles » (connues comme telles ou attribuables au travail ou à la supervision d'un sculpteur Tarawhai unique, parfois anonyme), les quatre derniers chapitres (16-19, pp. 258-305) tentent de mesurer les changements survenus dans cet art d'abord du point de vue des motifs, des formes et des matériaux (un véritable changement " de langue », résume excellemment R. Neich), mais aussi dans les relations avec les commanditaires, et enfin dans les représentations mêmes que se sont successivement formées les sculpteurs Tarawahai de leur art et de leurs créations. Si la conclusion (pp. 306-308) appelant à de nouvelles recherches sur le sujet fait apprécier toute la modestie du savant, sa générosité à livrer les connaissances qu'il a recueillies, et son souci de les rendre facilement accessibles s'observent de nouveau avec ses neuf appendices (pp. 309-402), alternant précieux inventaires, développements historiques et thèmes de recherches impossibles à détailler ici, et avec les six index dont il a tenu à munir son livre (pp. 415-424).

Imprimé en petit corps, illustré de photographies que le lecteur renonce à compter tant elles se succèdent (parfois 6 par page) et sont inséparables des argumentations de R. Neich, cet épais volume est à ranger au nombre des classiques de l'histoire de l'art, non seulement pour l'abondance exceptionnelle de données et de réflexions qu'il offre au public en des termes la plupart du temps accessibles à tous, mais aussi pour sa méthode, qui a orienté et semble résumer le parcours de ce savant, réussissant à élargir sans cesse ses investigations et ses thèmes de recherche depuis ses premières enquêtes dans la région de Rotorua, et tenant en même temps à approfondir celles-ci jusqu'à ses dernières années. Mais est-ce là signaler cette publication d'un point de vue suffisamment « scientifique » et « critique » comme il est attendu dans ces colonnes ? Deux remarques, étayées sur des autorités dont $\mathrm{R}$. Neich fut le contemporain et devint rapidement l'égal, suffiront peut-être à dissiper ce genre de doute.

Terence Barrow (1923-2001), dans des publications qu'on ne saurait énumérer ici, a constamment signalé combien la dimension "érotique » des objets d'art polynésiens (et particulièrement māori dont il était grand spécialiste) s'était trouvée minorée ou occultée par les premiers Occidentaux qui mirent la main dessus pour les détruire ou les exporter (avec mutilation des sculptures), ou en firent exécuter des répliques plus conformes à la «pudeur». R. Neich aborde ce sujet (qui doit aussi être envisagé sous maints autres angles, familial, généalogique, politique...) avec une prudence et une rigueur qui appellent l'admiration, notamment à propos du motif des " couples enlacés 》 (embracing figures, pp. 280-282 pour le texte, pp. 280-285 pour les illustrations, et inventaire de ces figures dans l'appendice VIII pp. 396-398), dont il montre qu'il offrit surtout aux maîtres sculpteurs Tarawhai l'occasion de déployer leur virtuosité en expérimentant vues frontales ou de profil, membres ou parties du corps en commun, et figurations encore plus « ambiguës », amusantes ou déroutantes.

Un autre grand spécialiste des arts māori dont Carve d Histories conduit à évoquer les travaux, David R. Simmons, à qui R. Neich succéda comme conservateur des collections ethnologiques du musée d'Auckland, s'était attiré maintes critiques pour son empressement à assigner les objets à des styles régionaux précis en s'appuyant principalement sur leurs caractéristiques formelles, parfois arbitrairement ou confusément définies. Que cette tâche soit des plus difficiles, R. Neich le soulignait lui-même (1996, pp. 94-97) en faisant observer que dès la fin du XVIII ${ }^{\mathrm{e}}$ siècle apparaissent des objets où se mêlent plusieurs styles locaux, probablement du fait de l'intrusion des Occidentaux, surtout dans les régions côtières de l'île du Nord, celles des Arawa et des Tarawhai particulièrement. Le cheminement de ce volume suffit à l'indiquer, R. Neich n'a nullement rejeté l'ambition affichée par D. R. Simmons après d'autres (Best, Skinner, etc.), il lui a apporté la perspective et la rigueur historiques qui lui manquaient jusqu'alors. Sous cet aspect, qui fait pleinement comprendre le titre de Carved Histories, ce livre restera aussi comme une leçon de méthode exemplaire et comme un grand livre d'histoire.

\section{RÉFÉRENCES CITÉES}

NeICH Roger, 1996. Wood-carving, in D. C. Starzecka (ed.), Maori Art and Culture, London, British Museum Press, pp. 69-113.D

-, 2008. A recently revealed tino-aitu figure from Nukuoro Island, Caroline Islands, Micronesia, Journal of the Polynesian Society 117-4, pp. 327344.

NeICH Roger and Stuart PARK, 2009. A detailed provenance for Kawe, the Nukuoro figure carving in Auckland Museum, Journal of the Polynesian Society 118-4, pp. 369-375.

Gilles BOUNOURE

Starzecka Dorota C., Roger NeICH and Mick Pendergrast, 2010. Taonga Mãori in the British Museum, Wellington, Te Papa Press, viII-168 pages, appendices, bibliogr., index, 2 cartes, 24 planches couleur, 200 planches noir et blanc, autres ill. noir et blanc dans le texte.

Ni Roger Neich ni Mick Pendergrast, tous deux décédés en 2010, n'auront eu en mains ce livre, sorti 
des presses au début de l'année suivante, selon le communiqué de presse annonçant sa parution. Ils avaient entrepris cette somme avec Dorota Starzecka au terme de la belle exposition « Maori Art and Culture » organisée par cette dernière en 1998 au British Museum. Ne fallait-il pas consacrer un "catalogue définitif » aux richissimes collections māori de ce musée ? Ils s'y sont appliqués pendant plus d'une décennie, et l'ouvrage qui en résulte est évidemment indispensable aux spécialistes, autant que le demeure, malgré ses défauts, le catalogue dressé par David Simmons pour les musées nord-américains (curieusement absent de la bibliographie du volume). Mais cette publication s'adresse aussi à d'autres lecteurs, les Māori euxmêmes en premier lieu, et, espère également le directeur du British Museum, les visiteurs de ces collections, parmi les plus fréquentées de son département dévolu aux civilisations d'Afrique, d'Océanie et des Amériques.

Après une introduction offrant de très précises indications sur l'histoire des collections (pp. 5-24) complétées en milieu de volume par une liste alphabétique des principaux «collectors, donors, vendors, institutions » (pp. 146-149, avec renvois à la bibliographie), douze sections (1. canots et accessoires, 2. greniers, maisons de réunion et sculptures diverses, 3. objets domestiques, 4 . boîtes à trésor, ornements et instruments de tatouage, 5. jeux et instruments de musique, 6. objets cérémoniels, mémoriels et mortuaires, 7. armes, 8. instruments de pêche, de chasse et d'agriculture, 9. outils de tissage et textiles, 10. céramiques modernes, 11 . objets des îles Chatham, 12. objets ou répliques de style māori) forment le catalogue proprement dit, avec un parti pris typologique des plus utiles, notamment pour l'étude des formes et des techniques. Des appendices sur l'architecture, les motifs sculptés et tissés, les techniques de tissage, un glossaire et cinq index ferment le volume.

2351 spécimens se trouvent ainsi non pas seulement décrits et photographiés, mais étudiés et réellement discutés dans des notices remarquables de concision et de franchise. D. Starzecka et R. Neich divergent fréquemment sur les datations assignables aux objets : par exemple, sur les dix-huit casse-tête en pierre (ou patu onewa) que mentionne la page 84 ( $\mathrm{n}^{\circ} 759$ à 776), huit que la première attribue au XVIII ${ }^{\mathrm{e}}$ siècle sont, pour le second, des productions du siècle suivant, mais on observe non moins souvent que la première date du $\mathrm{XIX}^{\mathrm{e}}$ siècle des pièces jugées par le second d'une époque antérieure (voir par exemple pp. 46-47 à propos des wakahuia ou boîtes à trésor). S'étendant parfois aussi aux régions où ces objets furent présumablement créés, ces divergences ne donnent que plus de poids aux points d'accord entre ces éminents spécialistes, tout en montrant sans faux unanimisme quels progrès a encore à accomplir la connaissance des arts māori, malgré les avancées considérables qu'elle a connues ces dernières décennies.

Ce catalogue, prévient l'éditeur, ne recense ni « os non travaillés », ni « pierres sculptées par le vent », ni pièces entrées dans les collections depuis 2000. On y relève également l'absence, sans commentaire ni justification, de toute tête humaine tatouée et momifiée māori (toi moko ou mokomakai), alors que le British Museum, au moment de la parution du volume, ne s'était toujours pas engagé dans la procédure de restitution de ces objets à la Nouvelle-Zélande, probablement pour ne pas avoir à restituer d'autres pièces qui lui sont réclamées de longue date, en Europe notamment (Peltier et Mélandri, 2012: 30). Il conviendra de se reporter à leur sujet à d'anciennes publications comme celle de Robley (1896 : 190 sq. pour les mokomakai du British Museum). On y trouve néanmoins reproduits, décrits et commentés d'assez nombreux objets incluant des restes humains, à l'instar des pendentifs hei tiki en calotte de crâne humain (p. 49, pl. 69), des pendants d'oreille et colliers de dents humaines (pp. 58-59 et pl. 79-80), des flûtes nasales en os humain, koauau (p. 65 et pl. 90), etc.

L'intérêt d'une aussi volumineuse publication prêtera peut-être à discussion en un temps où le British Museum, comme d'autres grands musées, s'est doté d'une base de données remarquable, sans cesse enrichie et corrigée, offrant souvent plusieurs vues en couleur du même spécimen, et permettant de visiter le meilleur de ses collections à distance. Mais près des deux tiers de l'humanité ne disposent toujours pas d'accès à internet, et pour le tiers plus favorisé, il n'est pas rare que les disparités d'équipement et de « débit » entre pays, voire entre villes d'une même région, empêchent de consulter utilement ces bases de données institutionnelles, comme on l'observe même depuis certaines zones insuffisamment «connectées" de France et d'Europe où la documentation imprimée demeure aujourd'hui irremplacée.

Ce sont néanmoins les mieux traités de ces privilégiés, accédant sans difficulté à l'excellente «British Museum collection database ", qui pourront mesurer ce qu'a de proprement irremplaçable le présent catalogue imprimé, à la fois pour le travail colossal de classement typologique détaillé qui s'y trouve condensé, " tâche " hors de portée des bases de données même munies des « filtres » les plus sophistiqués, et pour l'organisation de ses 200 planches en noir et blanc, permettant parfois de comparer jusqu'à 8 ou 9 objets par page, avec une facilité et une rapidité de consultation qu'on ne saurait attendre d'un nombre équivalent d'écrans. Il se pourrait même que les avantages très consistants des catalogues électroniques ne puissent être réellement exploités qu'à partir d'ouvrages imprimés aussi intelligemment et sérieusement conçus que Taonga Māori.

\section{RÉFÉRENCES CITÉES}

Peltier Philippe et Magali Mélandri, 2012. Chronologie concernant les têtes tatouées et momifiées māori ou toi moko, Journal de la Société des Océanistes 134 , pp. 28-30.

Robley Major-General, 1896. Moko or Maori Tattooing, London, Chapman and Hall.

Simmons David R., 1982. Catalogue of Maori Artefacts in the Museums of Canada and the United 
States of America, Auckland, Auckland Institute and Museum.

Starzecka Dorota C. (ed.), 1996. Maori Art and Culture, London, British Museum Press.

Gilles BounOuRE

CAmour Luc Enoka, 2010. Lien et séparation familiale de l'enfant kanak dans le monde socioculturel de Kaala-Gomen, préface d'Hamid Mokaddem, Pouembout, Éditions de la province Nord, 66 p.

Ce petit ouvrage de 66 pages fait le point un sujet qui fait couler beaucoup d'encre en Nouvelle-Calédonie depuis des années: l'enfant kanak et l'école ou le pourquoi de ses échecs ou réussites... Mais ici, il est le fait d'un Kanak originaire de Pouébo, enseignant qui plus est (à l'Alliance scolaire à Kaala-Gomen) et spécialisé depuis plusieurs années ${ }^{2}$ dans la remédiation et intervenant auprès des élèves en difficulté. Il sait donc de quoi il parle. Il veut réconcilier la famille et l'école, pensant que pendant trop longtemps, par le fait de privilégier la culture de l'école au détriment de celle de l'entant, on a coupé les familles de l'institution scolaire pour laquelle il éprouve souvent de la méfiance. Pour ce faire, l'auteur dans ce livre " décrit et explique le parcours typique d'un enfant kanak de la tribu dans son cheminement singulier vers le savoir universel de l'école » (p. 15).

Dans le chapitre 1, « Le milieu socioculturel kanak de Kaala-Gomen » (pp. 17-31), Luc Enoka Camoui propose un panorama de l'environnement socioculturel du village de Kaala-Gomen (situé sur la côte Ouest au Nord de la Grande Terre) et de ses tribus. Puis il dresse les différentes « carences induites ou produites par les rapports des enfants au milieu » (p. 20) : les carences d'ordre structurel (déstructuration familiale et sociale avec l'importance des jeunes mères célibataires...), celles d'ordres éducationnel et affectif. Il expose ensuite les « effets induits du trop de lien » (p. 27) avec le rôle de la famille, du clan et de la tribu dans le positionnement et la définition de l'enfant kanak où parfois «l'enfant hyper protégé ne parvient plus à conquérir le monde extérieur » (p. 29). Puis, l'analyse des résultats scolaires du collège de Kaala-Gomen de 1986 (date d'ouverture) à «maintenant » (20092010 ?) montre un taux de réussite des élèves de plus de $50 \%$ grâce aux élèves venant de Poum, Pouébo, Voh, Koné, mais pas pour les élèves habitant la commune qui ne parviennent pas à l'obtention du brevet des collèges. Étant « proches » du collège, ils sont externes et ne bénéficient donc pas de soutien scolaire pour les devoirs à faire (p. 30). Ici aussi, les enfants en échec reproduisent celui passé de leurs parents. Ce qui amène L. E. Camoui à conclure cette partie par cet énoncé implacable :
« Il serait souhaitable que l'enfant soit séparé momentanément de son espace familial pour vivre pleinement son cursus scolaire avec un encadrement qualifié de sorte qu'il fasse abstraction de cette mentalité réductrice qu'incarnent les parents. C'est à croire qu'à Kaala-Gomen, on vaccine très tôt les enfants contre la réussite scolaire [...]» (p. 31)

Dans le chapitre 2, «Séparation familiale ou le passage de la personne privée à la personne sociale » (pp. 33-51), l'auteur propose «sa solution» pour remédier à l'échec scolaire : acculturer l'enfant kanak en allant « au contact de l'autre » en se laissant :

« découvrir, [...] cannibaliser par l'autre afin de créer un monde symbiotique où la socialisation favorise l'émergence d'une culture syncrétique, enrichissante et qualifiante, purement virtuelle tendant à obliger l'enfant à s'individualiser pour être accepté et reconnu par ses pairs dans cette société artificielle mais si nécessaire où l'esprit de concurrence et de compétition est tel que l'enfant doit impérativement faire abstraction de ses mœurs, de ses tribulations pour s'insérer dans les normes de la culture de l'école, voir[e] même de l'universalité. » (p. 36)

Cette position peut paraître déroutante pour ceux qui comme moi ont connu la grande époque des événements des années 1980 et des revendications indépendantistes kanak avec notamment les écoles populaires kanak dont la position était me semble-t-il l'exacte inverse : pour dire vite, l'échec des enfants était dû au fait que ceux-ci étaient coupés de leur milieu social par l'école et il fallait donc les scolariser dans leur milieu et dans leur langue maternelle ! Il est sûr que le but de l'auteur est de voir « comment articuler les trajectoires d'élèves kanak avec les contraintes des trajectoires scolaires normalisantes » (p. 8). Mais finalement, la position de l'auteur n'est pas si éloignée par certains côtés de celles des Écoles populaires kanak (EPK) de l'époque (nous le verrons d'ailleurs plus précisément dans sa conclusion) puisqu'il reprend plus loin Pierre Bourdieu pour dire « qu'éduquer, c'est développer une intelligence historique capable de savoir dans quels héritages culturels on s'inscrit : de qui suis-je le fils ou la fille ? (p. 40). Ainsi, il tente de concilier toutes ces positions sur l'école :

«Sans parler de trahison culturelle, se séparer pour apprendre c'est toujours transgresser les règles et les idées reçues parce qu'apprendre c'est faire siens des savoirs pour se construire sa propre vision du monde, pour se situer au monde sans être dans la simple "reproduction". Du reste, apprendre, c'est se donner les outils pour relier de façon singulière ce qui est de l'ordre de l'universel, du culturel, afin de pouvoir créer sa propre autonomie aux rapports que l'on entretient avec l'autre, car il n'y a d'autonomie que dans la mesure où il y a dépendance. » (p. 41)

Sur le passage de la personne culturelle à la personne sociale (pp. 42-44), il présente des témoignages de Kanak ayant " une situation sociale confortable » qui insistent sur le fait que :

2. Auparavant, il a été éducateur à l'internat de Do Neva à Houaïlou, a préparé un diplôme d'anglais à l'université de Wellington (Victoria) pour être traducteur bilingue à Suva (Fidji). Il a aussi occupé divers postes dans l'enseignement primaire et dirigé durant près de quinze ans une école à Yambé avant de suivre une formation en intégration scolaire à Paris pour devenir maître spécialisé « Aide à l'intégration scolaire » (AIS) à Kaala-Gomen. 